\title{
Study of Probes and Substrates for Low Temperature Atomic Force Microscopy and Biological Applications
}

\author{
A. Radenovic* ${ }^{*}$ E. Bystrenova, L. Libioulle \\ AND G. DieTLer \\ Institute of Condensed Matter Physics, University of Lausanne \\ 1015 Lausanne, Switzerland
}

\begin{abstract}
The atomic force microscopy in ultrahigh vacuum and at low temperature demonstrated its excellent capability to reach atomic resolution. Nevertheless in the case of biological samples high resolution has been achieved only in very few cases. We demonstrated here the importance of the appropriate choice of probes and substrates in order to image DNA at low temperature with high resolution. We investigated properties of three types of cantilevers and they were studied by scanning electron microscopy as a function of temperature. A large bending of cantilevers, which were coated from both sides, was observed at low temperatures. Therefore uncoated cantilevers are strongly recommended for low temperature applications. Different methods for immobilization of DNA on the substrate are examined at low temperatures. First images of linear DNA on graphite at $82 \mathrm{~K}$ under ultrahigh vacuum conditions are presented.
\end{abstract}

PACS numbers: 07.79.Lh, 68.37.Hk, 82.39.Pj, 07.20.Mc

\section{Introduction}

The atomic force microscopy (AFM), first developed by Binnig et al. [1], has become a powerful tool in biology. It can provide three-dimensional images of biological specimens in ambient, liquid or gas environments. Unlike other techniques, AFM can image samples with just minor preparation, e.g. without staining, coating etc., and over a large range of temperatures or in repetitive studies. The good resolution (in the nanometer range) allows imaging samples such as DNA

*corresponding author; e-mail: Aleksandra.Radenovic@ipmc.unil.ch 
molecules with details. However, high resolution imaging of DNA or evidence of DNA helicity has been only achieved in a very few cases [2]. As demonstrated by many studies, biomolecular complexes are too soft for achieving high resolution imaging at room temperature in contact mode. Indeed the deformation of flexible biomolecular structures is very large under the AFM tip and the cantilever undergoes also thermal motion. All these effects reduce the instrument resolution and in order to circumvent these limitations, measurements on biological materials can be performed at low temperatures. Therefore we constructed a low temperature AFM (cryo-AFM) for studying biological samples. A detailed description of the instrument was previously reported [3]. However there are a few aspects of instrumentation and sample preparation that need special care for being able to image biological samples with high resolution at low temperatures. At the same time, the properties of used cantilevers have to be known precisely. Many methods were developed so far to measure the relevant properties of specific cantilevers. In this paper, these methods are extended to low temperatures and ultrahigh vacuum (UHV) conditions.

\section{Experimental methods and results}

\subsection{Characterization of atomic force microscope probes at low temperatures}

The choice of the correct probe is crucial to work properly at low temperature with biological samples. Therefore we have characterized the three types of contact-mode triangular cantilevers that we have selected to use. We estimated first that operating at low temperature requires either cantilevers without any metallic coating or double-side coated cantilevers. The spring constants should also stay low because of the softness of biomolecules at room temperature. We need to obtain good images at room temperature prior to cool down the whole system for sample quality control. Therefore these requirements greatly restricted our choice of cantilevers. We investigated the selected cantilevers: the first two are both sides gold/chromium coated silicon nitride cantilevers (spring constants $0.02 \mathrm{~N} / \mathrm{m}$ and $0.08 \mathrm{~N} / \mathrm{m}$, Olympus OMLC-TR400 PB) and the third one is uncoated silicon cantilever (spring constant $0.12 \mathrm{~N} / \mathrm{m}$, MicroMasch CSC21).

Thermal noise spectra and force distance curves were measured for each type of cantilever. Figure 1a shows the thermal power spectra for the uncoated silicon cantilever as a function of temperature. The temperature dependence of the resonance frequency is shown in the corresponding inset. The resonance frequency, in air and at room temperature, was measured to be $4 \pm 0.5 \%$ different from the related value in vacuum. This is in agreement with previously reported measurements $[4,5]$. We have observed the temperature frequency shift to be $3-4 \%$ for all types of cantilevers [6], which can be explained by a change of Young's modulus of material as confirmed by a theoretical model in [5].

A force-distance curve is a compact way of representing the interaction between the probe and the sample and it allows a better understanding of AFM 


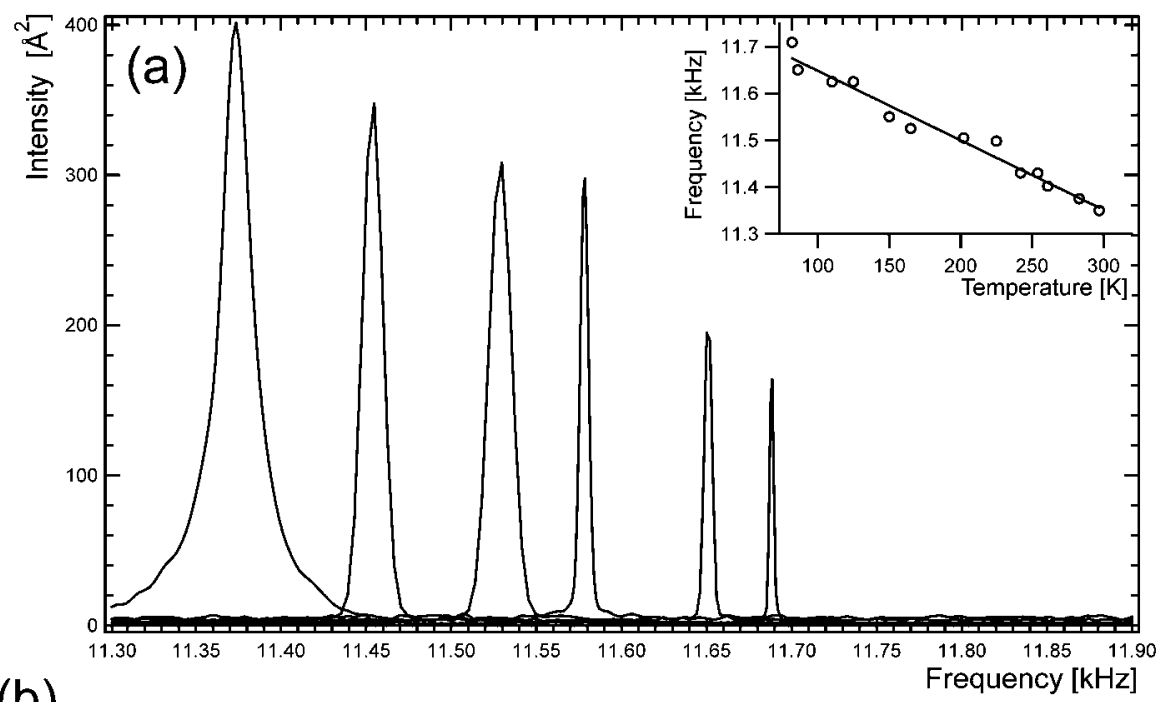

(b)

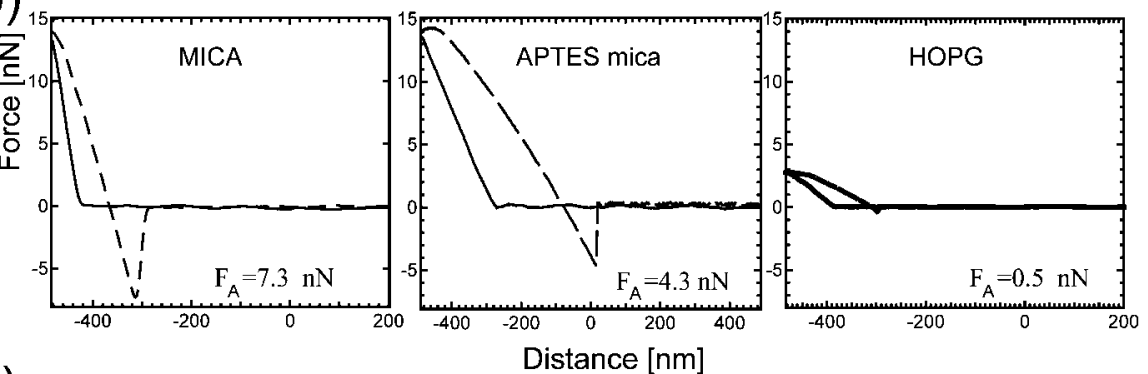

(c)
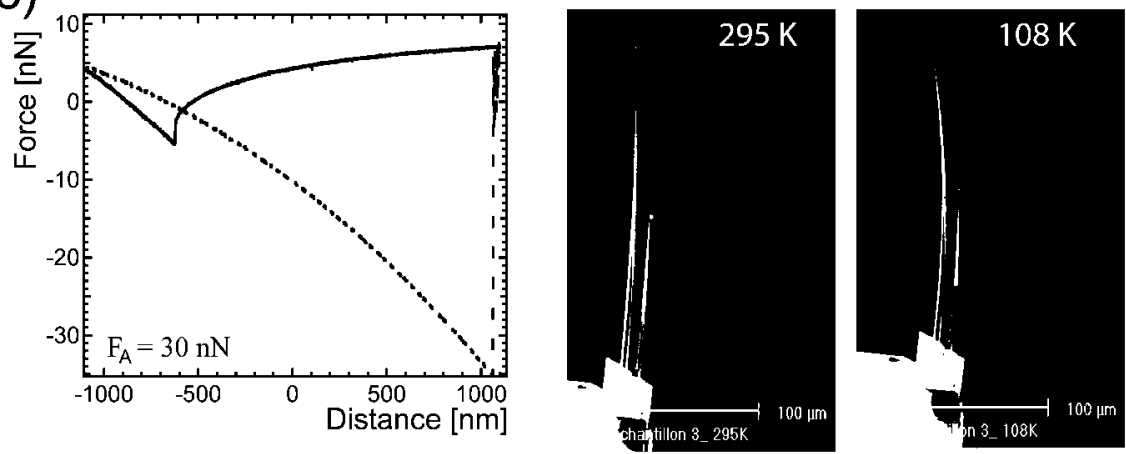

Fig. 1. (a) The thermal noise spectra acquired at different temperatures $(295 \mathrm{~K}, 240 \mathrm{~K}$, $200 \mathrm{~K}, 150 \mathrm{~K}, 110 \mathrm{~K}$, and $82 \mathrm{~K}$ from left to right) for uncoated silicon cantilever. The inset shows the temperature dependence of its resonance frequency. (b) Typical force distance curves of uncoated silicon cantilevers on mica, APTES mica, and HOPG at $82 \mathrm{~K}$ with the values of adhesion forces. (c) Force distance curve of gold/chromium coated silicon nitride cantilever on mica at $82 \mathrm{~K}$ and its micrographs at different temperatures are shown. 
images. In order to characterize an uncoated cantilever we performed a series of force distance curves on different substrates at $82 \mathrm{~K}$. They were recorded using a LabVIEW program (National Instruments) and they are shown in Fig. 1b.

Freshly cleaved mica and graphite (highly ordered pyrolytic graphite (HOPG)) substrates were used. After cleavage, mica is known to carry some negative surface charges. They can induce opposite charges in the gold/chromium coating of the cantilever material, and result in a large attractive force. This behavior is confirmed by long distance force, acting on the cantilever prior to the tip engagement; the force reaches therefore unusual large negative values as shown in Fig. 1c. A proper grounding of the specimen may lower this adhesion force. On the other hand, the graphite substrate is conductive and the surface charges can be totally canceled while connecting the sample properly to the ground.

The next characterization of the probes was performed by scanning electron microscopy (SEM) in order to directly observe the bending of coated cantilevers. Experiments were performed using a Philips XL-30 SFEG microscope operating at $5 \mathrm{kV}$ and coupled with a cryotransfer (Gatan Alto 2500). The micrographs obtained at room temperature and at $108 \mathrm{~K}$ are shown in Fig. 1c. An apparent bending of double sides coated cantilever is visible at low temperature. This bending can be explained by difference of a few nanometers in the thickness of two layers, which cantilever was coated with. The bending contributes also to the atypical shape of its force distance curve shown in Fig. 1c. The proper use of uncoated cantilevers is confirmed since no bending effect is observed. This is the most appropriate type of cantilever for operation at low temperature [6].

\section{Sample preparation}

The driving force for studies on DNA lies in potential high-resolution of the AFM imaging. On the other hand, nearly all AFM measurements on DNA have shown a much reduced height in the range of $1-1.8 \mathrm{~nm}[7,8]$ compare to the X-ray crystallographic data [9]. At a temperature close to $180 \mathrm{~K}$, the structural rigidity of most macromolecules has been shown to be much stiffer [10] and the adhesion to the substrate can be improved. We extended the different DNA adsorption techniques known to work well at ambient conditions, to low temperatures application.

Three different types of DNA have been investigated. Plasmid DNA was a gift from Dr. Viglasky (Dep. of Biochemistry, University of Kosice), the protocol of purification is described in [11]. DNA knots were kindly supplied by the laboratory of Dr. Roca (Instituto de Biologia Molecular de Barcelona) and are extracted from bacteriophage P4 capsids [12]. Lambda mix linear DNA has been purchased from MBI FERMENTAS (St. Leon-Rot, Germany).

The DNA molecules should be adsorbed on substrates which have flat surfaces at atomic level such as mica or HOPG. The surface characteristics of these substrates are different and therefore they yield different deposition protocols. 


\subsection{Adsorption of DNA on mica in the presence of divalent cations}

Mica, the most common substrate for AFM, consists of tetrahedral double sheets of $(\mathrm{Si} / \mathrm{Al})_{2} \mathrm{O}_{5}$ electrostatically linked by potassium ions. This electrostatic interaction plays an important role for adsorbing and binding DNA. By improving, the method of ionic treatment of mica [13], suggested earlier for sample preparation used in transmission electron microscopy, one can use divalent cations (e.g. $\mathrm{Mg}^{2+}$, $\mathrm{Zn}^{2+}$ ) to increase the mica affinity to DNA. Later according to [7], untreated mica was found also to be usable, while depositing DNA with a magnesium salt. It is not clear whether these cations act by directly binding of DNA to the charged mica substrate, altering the surface charge, or electrical double layer formation, promoting the adsorption and/or inhibiting the desorption. However, this method proved to be stable enough and good quality images at ambient conditions can be easily obtained.

We have imaged DNA plasmids adsorbed in the presence of divalent cations on freshly cleaved mica by cryo-AFM at different temperatures, Fig. 2a. The lowest temperature at which we were able to obtain high resolution images of DNA plasmids was $180 \mathrm{~K}[3]$. Consecutive, while approaching temperature towards $82 \mathrm{~K}$,
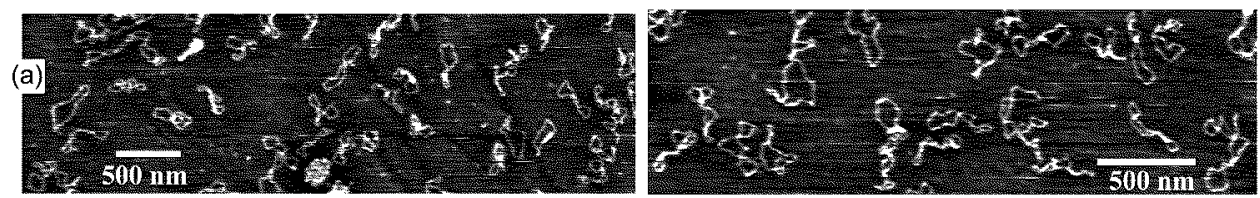

(b)
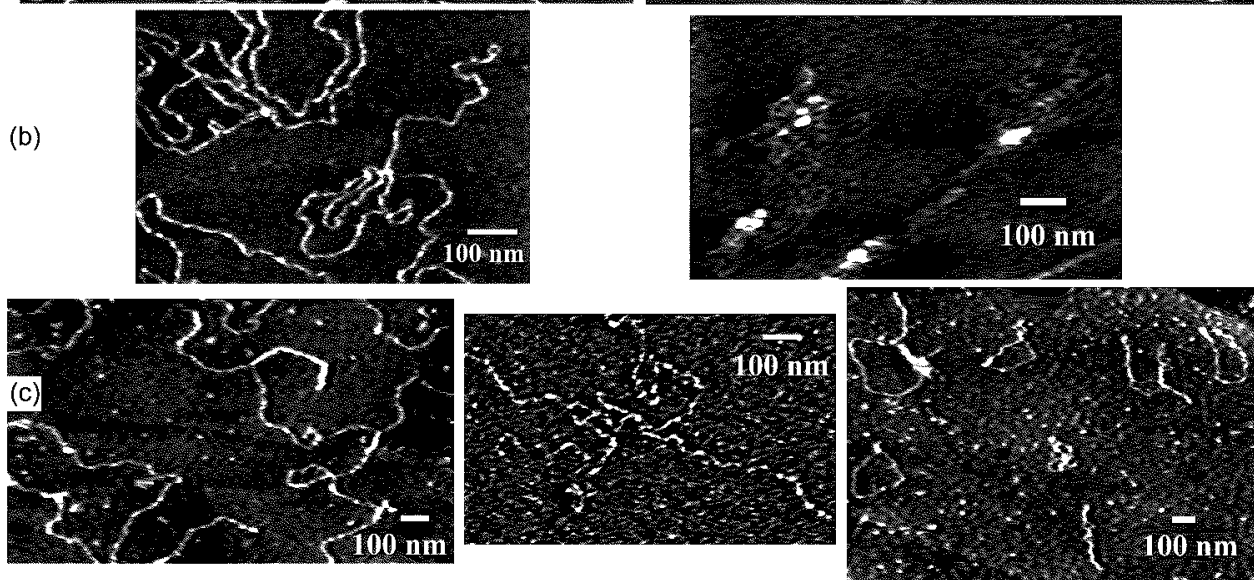

Fig. 2. (a) The images of DNA plasmids deposited on mica surface in presence of divalent cations at ambient conditions (left) and at $180 \mathrm{~K}$ (right). (b) The image of linear DNA deposited on APTES mica surface at ambient conditions (left) and image of DNA knots on APTES mica at $200 \mathrm{~K}$ (right). (c) The image of linear DNA on HOPG at ambient conditions (left), DNA plasmids on HOPG at $200 \mathrm{~K}$ (right) and inset in the middle shows linear DNA at $82 \mathrm{~K}$. All images were obtained in contact mode with cryo-AFM. 
a certain contamination in the range of 4-5 $\mathrm{nm}$ appeared on the mica surface and imaging of DNA turn to be impossible. The formation of a water layer on the hydrophilic mica surface was addressed previously in the literature $[14,15]$. This could be one of the reasons of high adhesion we have observed at that temperature, as shown in Fig. 1c. Accordingly, the proper choice of hydrophobic substrates for working at low temperature was essential. However images obtained in contact mode with cryo-AFM at $180 \mathrm{~K}$ shows already improvement in the height of DNA as it was reported previously in [3].

\subsection{Adsorption of DNA on the functionalized (APTES) mica surface}

It is known that silanized surface of mica is slightly hydrophobic. Authors of [16] have implemented a new method for the strong adsorption of DNA on mica. Their method, for chemically modifying mica substrates, uses covalently bound 3-aminopropyltriethoxysilanehyl (APTES) followed by methylation and hydrolysis. In liquid, the positively charged amine groups bind negatively charged DNA. This good adsorption provides excellent AFM imaging conditions. The higher hydrophobicity of APTES mica, in comparison with mica, was confirmed by cryo-AFM, since contamination on the sample surface was only in the range of $1-1.5 \mathrm{~nm}$ at $82 \mathrm{~K}$. Unfortunately, in these conditions scanning of DNA was still difficult. The images obtained in contact mode with cryo-AFM at ambient conditions and at $200 \mathrm{~K}$ are shown in Fig. $2 \mathrm{~b}$.

\subsection{Adsorption of DNA on HOPG surface}

Another substrate commonly used in scanning tunneling microscopy is graphite. It seems suitable for biological imaging and it is strongly hydrophobic. Proper grounding can be realized with conducting graphite very easily. Therefore we chose this substrate for imaging by AFM at low temperatures although the process of DNA adsorption to graphite is more complicated than in the case of mica.

Immobilization by free adsorption is the simplest way to attach nucleic acid to a surface but the HOPG surface possesses hydrophobic characteristics and DNA is a highly charged molecule, consequently the capacity of spontaneous interaction of DNA with the HOPG surface should be reduced.

DNA was adsorbed freely at the HOPG surface and tapping mode AFM images in air were performed. The affinity of the hydrophobic DNA bases to interact with the HOPG stabilizes the DNA at the surface [17], but DNA molecules deposited in this way do not adhere sufficiently to the surface to be imaged in contact mode AFM.

It is well documented [18] that the HOPG surface treated by glow discharge technique possesses polar surface groups. The existence of polar groups renders the surface of HOPG into temporarily hydrophilic. The surface of HOPG was treated for 10-30 seconds in oxygen plasma. After glow discharge treatment with oxygen 
plasma, the HOPG is made hydrophilic and negatively charged, thus allowing an easy spreading of DNA suspension. With subsequent magnesium acetate or magnesium chloride treatment the surfaces were made hydrophilic and positively charged (as in case of mica) and after a brief time it recovers its hydrophobicity. The DNA molecules adhered better to such surface and the good adsorption allows contact mode AFM imaging. The drawback of this method is the higher roughness of the substrate which was found to be in the range of $0.2-0.5 \mathrm{~nm}$. In Fig. $2 \mathrm{c}$ images of linear DNA and DNA plasmids obtained in contact mode with cryo-AFM at ambient conditions, at $200 \mathrm{~K}$ and at $82 \mathrm{~K}$, are shown. Recovered hydrophobicity of HOPG after its treatment was confirmed while imaging DNA at $82 \mathrm{~K}$ where no contamination was observed. The lower adhesion of HOPG compared to mica or APTES mica at $82 \mathrm{~K}$ is shown in Fig. 1b which demonstrates the proper choice of this substrate.

\section{Conclusion}

Property of AFM probes, such as the resonance frequency with a typical shift of $3-4 \%$ as a function of temperature was observed. The cantilevers were imaged by the cryo SEM at different temperatures and a bending of the coated cantilevers at low temperatures was observed. As a consequence, the use of uncoated cantilevers for low temperature applications is recommended. The different techniques of DNA adsorption, to mica, APTES mica, and graphite surface were investigated. We demonstrated importance of hydrophobicity of the substrate for high resolution imaging at low temperature and under UHV conditions. First images of linear DNA on HOPG obtained at $82 \mathrm{~K}$ are presented.

\section{References}

[1] G. Binnig, C.F. Quate, C. Gerber, Phys. Rev. Lett. 56, 930 (1986).

[2] H.G. Hansma, D.E. Laney, M. Bezanilla, R.L. Sinsheimer, P.K. Hansma, Biophys. J. 68, $1672(1995)$.

[3] A. Radenovic, E. Bystrenova, L. Libioulle, M. Taborelli, J.A. DeRose, G. Dietler, Rev. Sci. Instrum. 74, 1022 (2003)

[4] J.E. Sader, Rev. Sci. Instrum. 66, 4583 (1995).

[5] F. Giessibl in: Noncontact AFM, Eds. S. Morita, R. Wiesendanger, E. Meyer, Springer-Verlag, New York 2002, p 38.

[6] A. Radenovic, E. Bystrenova, L. Libioulle, F. Valle, G.T. Shubeita, S. Kasas, G. Dietler, J. Appl. Phys. 94, 4210 (2003).

[7] H.G. Hansma, I. Revenko, K. Kim, D.E. Laney, Nucl. Acids. Res. 24, 713 (1996).

[8] Z. Shao, J. Yang, A.P. Somlyo, Ann. Rev. Cell Dev. Biol. 11, 241 (1995).

[9] J.D. Watson, F.H.C.Crick, Nature 171, 737 (1953). 
[10] I.E.T. Iben, D. Braunstein, W. Doster, H. Frauenfelder, M.K. Hong, J.B. Johnson, S. Luck, P. Ormos, A. Schulte, P.J. Steinbach, A.H. Xie, R.D. Young, Phys. Rev. Lett. 62, 1916 (1989).

[11] V. Viglasky, M. Antalik, J. Adamcik, D. Podhradsky, Nucl. Acids. Res. 28, e51 (2000).

[12] S. Trigueros, J. Arsuaga, M.E. Vazquez, D.W. Sumners, J. Roca, Nucl. Acids. Res. 29, e67 (2001).

[13] M. Bezanilla, S. Manne, D.E. Laney, Y.L. Lyubchenko, H.G. Hansma, Langmuir 11, 655 (1995).

[14] S. Park, G. Sposito, Phys. Rev. Lett. 89, 085501 (2002).

[15] C.B. Prater, M.R. Wilson, J. Garnaes, J. Massie, V.B. Elings, P.K. Hansma, J. Vac. Sci. Technol. B 9, 989 (1991).

[16] Y. Lyubchenko, L. Shlyakhtenko, R. Harrington, P. Oden, S. Lindsay, Proc. Natl Acad. Sci. USA 90, 2137 (1993).

[17] D. Bensimon, A.J. Simon, V. Croquette, A. Bensimon, Phys. Rev. Lett. 74, 4754 (1995).

[18] J.V. Zoval, P.R. Biernacki, R.M. Penner, Anal. Chem. 68, 1585 (1996). 